\title{
Normal Forms in Function Fields
}

\author{
Karl Aberer, \\ ETH Zürich
}

\begin{abstract}
We consider function fields of functions of one variable augmented by the binary operation of composition of functions. It is shown that the straightforward axiomatization of this concept allows the introduction of a normal form for expressions denoting elements in such fields. While the description of this normal form seems relatively intuitive, it is surprisingly difficult to prove this fact. We present an algorithm for the normalization of expressions, formulated in the symbolic computer algebra language mathematica. This allows us to effectively decide compositional identities in such fields. Examples are given.
\end{abstract}

\section{Introduction}

One of the fundamental questions in symbolic computation is to find normal forms for expressions where certain identities are considered to be valid for these expressions. Function fields are structures appearing frequently as domains for symbolic computations, one has to think only of the rational functions, elementary functions, special functions etc. . Such function fields satisfy, besides the identities resulting from their property of being a field, additional identities resulting from the properties of the composition operator. These properties are known very well, and important contributions to the investigation of spaces equipped with composition as an additional operation were especially made by K. Menger [6]. It now seems natural to ask for normal form algorithms in such spaces, in our case function fields, to be able e.g. to computationally decide the equivalence problem for terms. In the following example let $a, F, j$ be unary function symbols, where addi- tionally $j(x)=x$, for all $x$. It is quite straightforward to simplify the term

$$
\left((F+1)\left(1+a\left((j+1)^{2}\right)\right)\right)(x-1)
$$

to

$$
F\left(1+a\left(x^{2}\right)\right)+1
$$

But first how do we know that this is the simplest or an otherwise outstanding form of that term and second what to do when the terms get longer and computation by hand gets error prone or even impracticable? For example: does the following expression have a simple equivalent "normal" form:

$$
\begin{aligned}
& \left(\left((F+1)\left(1+a\left((1+j)^{2}\right)\right)\right)(a-1)+\right. \\
& \left.j \cdot\left(1+F\left(1+a\left(a^{2}\right)\right)\right)\right)\left(F\left(a+a^{2}\right)\left(x^{2}-1\right)-\right. \\
& \left.\quad(F+1)\left(a\left(j^{2}-1\right)(x) \cdot(1+a(j \cdot(2+j))(x-1))\right)\right) .
\end{aligned}
$$

(with multiplications indicated by ·.) When expressions get a certain size, and as we will see the normal forms can grow exponential in length, the help of computers is unavoidable. With the help of the computer algebra system mathematica we will be able to implement the normal forms for expressions like the ones given above. The goal of the algorithm is to reduce every term to a simple form of which we can show by construction of an appropriate substitution of the function symbols that it is not identical to zero iff the expression itself is nonzero. This form is the desired normal form.

This work is part of a project to develop a system to compute in combinatory analytic structures and languages, e.g. fields or vector spaces with operators appropriate to represent programs. For a short motivation compare with section 6 where the terms of the first of the two examples above are interpreted as programs. Extensions of our algorithm to the case where differentiation (differential fields) or programming constructs like if..then..else are involved were made [1] and are used as a part of a system to compute in combinatory differential fields [3]. 


\section{Definitions}

First we give a general definition of the notion of normal form. Let $T$ be a set of terms and $=_{T}$ an equivalence relation on the terms.

Definition 1 (Normal form) A normal form is given by a normal form function

$$
N: T \rightarrow N T \subseteq T, \quad \tau \mapsto N(\tau),
$$

with the properties

$$
\begin{array}{ll}
\text { 1. } & N(N(\tau)) \equiv N(\tau), \\
\text { 2. } & a={ }_{T} b \text { iff } N(a) \equiv N(b) .
\end{array}
$$

The relation $\equiv$ means syntactically equal. The set NT is the set of terms in normal form.

Next we define a language $\mathcal{C}$ in which we describe the properties of function fields and for whose terms we intend to construct normal forms. The terms are built up from the individual constants 0,1 and $\iota$, variables $x_{1}, x_{2}, x_{3} \ldots$, the field operations $+, \cdot,-{ }^{-1}$, and the composition operation $\circ$. The predicates are $=$ and const. We characterize the structure of function fields by the following first order theory formulated in the language $\mathcal{C}$, using $\sigma, \tau, \ldots$ as metavariables for terms.

Definition 2 (Field axioms $A_{R}$ ) A function field is a field of characteristic 0 with neutral element 0 for addition and 1 for multiplication. A subset of elements $x$ with const $(x)$ true are called the constant elements and form a subfield.

$$
\begin{gathered}
\operatorname{const}(0), \operatorname{const}(1), \\
\operatorname{const}(\sigma) \wedge \operatorname{const}(\tau) \rightarrow \operatorname{const}(\sigma+\tau) \wedge \operatorname{const}(\sigma \cdot \tau), \\
\operatorname{const}(\tau) \rightarrow \operatorname{const}(-\tau), \\
\operatorname{const}(\tau) \wedge \tau \neq 0 \rightarrow \operatorname{const}\left(\tau^{-1}\right)
\end{gathered}
$$

\section{Definition 3 (Axioms of composition $A_{C}$ )}

$$
\begin{aligned}
& \left(\tau_{1}+\tau_{2}\right) \circ \sigma=\tau_{1} \circ \sigma+\tau_{2} \circ \sigma, \\
& \left(\tau_{1} \cdot \tau_{2}\right) \circ \sigma=\tau_{1} \circ \sigma \cdot \tau_{2} \circ \sigma, \\
& (-\tau) \circ \sigma=-(\tau \circ \sigma), \\
& \left(\tau^{-1}\right) \circ \sigma=(\tau \circ \sigma)^{-1}, \\
& \tau_{1} \circ\left(\tau_{2} \circ \tau_{3}\right)=\left(\tau_{1} \circ \tau_{2}\right) \circ \tau_{3}, \\
& \tau \circ \iota=\iota \circ \tau=\tau, \\
& \operatorname{const}(\sigma) \rightarrow \sigma \circ \tau=\sigma \text {. }
\end{aligned}
$$

We introduce different classes of terms.

\section{Definition 4 (Term classes)}

(1) Constant Terms Q: Terms built up with the constants 0,1 and operations $+, \cdot,-,{ }^{-1}$ containing no variables.

(2) Rational Terms $R\left(\sigma_{1}, \ldots, \sigma_{k}\right)$ : Terms built up with the constants 0,1 and operations $+, \cdot,-,-1$ using terms $\sigma_{1}, \ldots, \sigma_{k}$.

(3) Composition Terms $C\left(x_{1}, \ldots, x_{k}\right)$ : Terms built up with the constants $0,1, \iota$ and operations $+, \cdot,-,{ }^{-1}$, 。 using variables $x_{1}, \ldots, x_{k}$.

\section{Example 1}

$$
\begin{gathered}
R(\iota, x) \subseteq C(x) . \\
Q \subseteq R\left(x, x \circ x, x \circ\left(x^{2}\right), \ldots\right) \subseteq C(x) .
\end{gathered}
$$

On these classes we define the following equivalence relations, where we use "上" to denote provability in firstorder logic with equality.

\section{Definition 5 (Equivalence relations on terms)}

$$
\begin{aligned}
& \text { (1) } \tau={ }_{R} \sigma \text { for } \tau, \sigma \in C\left(x_{1}, x_{2}, \ldots\right) \text { iff } \\
& A_{R} \vdash \tau=\sigma . \\
& \text { (2) } \tau={ }_{C} \sigma \text { for } \tau, \sigma \in C\left(x_{1}, x_{2}, \ldots\right) \text { iff } \\
& \\
& A_{R} \cup A_{C} \vdash \tau=\sigma .
\end{aligned}
$$

Remark. Hence we have $\tau \neq{ }_{R} \sigma$ iff $A_{R} \nvdash \tau=\sigma$ and $\tau \neq_{C} \sigma$ iff $A_{R} \cup A_{C} \forall \tau=\sigma$. Our goal will be to construct a normal form function $N_{C}$ under the equivalence relation $=_{C}$.

\section{Rational Terms}

The obvious normal form for $Q$ under $={ }_{R}$ is given by:

$$
\begin{aligned}
N Q=\left\{\frac{p}{q} \mid p \equiv \pm(1+1+\ldots+1),\right. & \\
& q \equiv 1+1+\ldots+1, \operatorname{gcd}(p, q)=1, p, q \in \mathbb{Z}\} .
\end{aligned}
$$

Similarly for rational terms in the class $R\left(x_{1}, \ldots, x_{k}\right)$ a class of normal forms under $=_{R}$ (with $N P$ the class of normal forms of polynomials over $\mathbb{Z}$, i.e. normal forms for elements in $\left.\mathbb{Z}\left[x_{1}, \ldots, x_{k}\right]\right)$ is given by:

$$
\begin{gathered}
N R\left(x_{1}, \ldots, x_{k}\right)=\left\{\frac{p}{q} \mid p, q \in N P\left(x_{1}, \ldots, x_{k}\right),\right. \\
l c(q)>0, \operatorname{gcd}(p, q)=1\} .
\end{gathered}
$$

Normal forms for polynomials are trivial, one could take the polynomial expanded with equal powers collected and sorted in a canonical order. We want to summarize in the following lemma.

Lemma 1 For the class $Q$ there exists a normal form function $N_{Q}$ which computes the unique normal form for terms in $Q$ under the equivalence relation $=_{R}$. For the class $R\left(x_{1}, \ldots, x_{k}\right)$ there exists a normal form function $N_{R}$ which computes the unique normal form for terms in $R\left(x_{1}, \ldots, x_{k}\right)$ under the equivalence relation $={ }_{R}$. 


\section{Composition Terms}

Remark. To increase the readability we want to use in the following lower case latin letters for rational terms and lower case greek letters for terms in $C$. Nevertheless the type of a term always will be obvious from the context. In the proofs we therefore often suppress argument lists.

Lemma 2 For terms $\tau \in C$ and $t\left(\iota, x_{1}, \ldots, x_{k}\right) \in$ $R\left(\iota, x_{1}, \ldots, x_{k}\right)$ the following equation holds.

$$
t\left(\iota, x_{1}, \ldots, x_{k}\right) \circ \tau={ }_{C} t\left(\tau, x_{1} \circ \tau, \ldots, x_{k} \circ \tau\right) .
$$

Proof. Induction on the structure of the term $t$.

Remark. We will use the following obvious properties:

(1) If $\sigma={ }_{R} \tau$ then $\sigma={ }_{C} \tau$ and the contraposition, if $\sigma \neq_{C} \tau$ then $\sigma \neq_{R} \tau$.

(2) The converse we can only state for $\sigma \in Q$. If $\sigma \in Q$ and $\sigma \neq_{R} 0$ then $\sigma \neq_{C} 0$. This property follows immediately from the field axiom $\neg(1=0)$ and the fact that all constant terms in $Q$ have a normal form in $N Q$.

The first non-trivial problem is to specify a subclass of composition terms that will serve as normal forms. We propose the following definition.

Definition 6 (Classes of composition terms) Let $V\left(x_{1}, \ldots, x_{k}\right)$ and $T\left(x_{1}, \ldots, x_{k}\right)$ be defined recursively as follows.

$$
\begin{aligned}
& V_{0}\left(x_{1}, \ldots, x_{k}\right):=\left\{x_{1}, \ldots, x_{k}\right\}, \\
& T_{n}\left(x_{1}, \ldots, x_{k}\right):=\left\{t\left(\iota, v_{1}, \ldots, v_{l}\right) \mid\right. \\
& v_{1}, \ldots, v_{l} \in \bigcup_{j \leq n} V_{j}\left(x_{1}, \ldots, x_{k}\right), \\
& t\left.\in N R\left(\iota, v_{1}, \ldots, v_{l}\right)\right\}, \\
& V_{n+1}\left(x_{1}, \ldots, x_{k}\right):=\left\{x_{i} \circ \tau \mid i=1, \ldots, k,\right. \\
& \tau\left.\in T_{n}\left(x_{1}, \ldots, x_{k}\right), \tau \not \equiv \iota\right\}, \\
& V\left(x_{1}, \ldots, x_{k}\right):=\bigcup_{j \in \mathbb{N}} V_{j}\left(x_{1}, \ldots, x_{k}\right), \\
& T\left(x_{1}, \ldots, x_{k}\right):=\bigcup_{j \in \mathbb{N}} T_{j}\left(x_{1}, \ldots, x_{k}\right) .
\end{aligned}
$$

Remark. We have $T_{0} \subset T_{1} \subset T_{2} \ldots$ and $V_{0} \subset V_{1} \subset$ $V_{2} \ldots$

Lemma 3 If $v \in V_{n}\left(x_{1}, \ldots, x_{k}\right)$ and $\tau \in T_{m}\left(x_{1}, \ldots, x_{k}\right), \tau \not \equiv \iota$, then there exists a term $u \in V_{n+m+1}\left(x_{1}, \ldots, x_{k}\right)$ such that $v \circ \tau={ }_{C} u$.

Proof. Induction on the structure of $v$.

Base step. Let $v \in V_{0}, v \equiv x_{i}$. Then take $u: \equiv x_{i} \circ \tau \in$ $V_{m+1}$ for $\tau \in T_{m}$.
Induction step. Let $v \in V_{n}, n>0, v \equiv x_{i} \circ \rho \equiv$ $x_{i} \circ r\left(\iota, v_{1}, \ldots, v_{h}\right)$ with $r \in N R\left(\iota, v_{1}, \ldots, v_{h}\right)$ and $\rho \in$ $T_{n-1}$. Then $v_{1}, \ldots, v_{h} \in \bigcup_{j \leq n-1} V_{j}$. Furthermore let $\tau \in T_{m}, \tau \equiv s\left(\iota, w_{1}, \ldots, w_{l}\right) \in N R\left(\iota, w_{1}, \ldots, w_{l}\right)$. Then again $w_{1}, \ldots, w_{l} \in \bigcup_{j \leq m} V_{j}$. We observe $v \circ \tau={ }_{C}\left(x_{i} \circ\right.$ $r) \circ \tau={ }_{C} x_{i} \circ(r \circ \tau)$. By application of lemma 2 we get

$$
\begin{aligned}
v \circ \tau==_{C} & x_{i} \circ\left(r\left(\iota, v_{1}, \ldots, v_{h}\right) \circ \tau\right)={ }_{C} \\
& x_{i} \circ r\left(\tau, v_{1} \circ \tau, \ldots, v_{h} \circ \tau\right)==_{C} \\
& x_{i} \circ r\left(s\left(\iota, w_{1}, \ldots, w_{l}\right), v_{1} \circ \tau, \ldots, v_{h} \circ \tau\right)={ }_{C} \\
& x_{i} \circ t\left(\iota, w_{1}, \ldots, w_{l}, v_{1} \circ \tau, \ldots, v_{h} \circ \tau\right),
\end{aligned}
$$

where $t \in R\left(\iota, w_{1}, \ldots, w_{l}, v_{1} \circ \tau, \ldots, v_{h} \circ \tau\right)$. We take $u: \equiv$ $x_{i} \circ N_{R}(t)$ and then $u \in V_{n+m+1}$ because $w_{1}, \ldots, w_{l} \in$ $\bigcup_{j \leq m} V_{j}$ and $v_{1} \circ \tau, \ldots, v_{h} \circ \tau \in \bigcup_{j \leq n+m} V_{j}$, by induction hypothesis.

\section{Lemma 4 There exists a function}

$$
N_{C}: C\left(x_{1}, \ldots, x_{k}\right) \rightarrow T\left(x_{1}, \ldots, x_{k}\right)
$$

such that for every composition term $\tau \in C\left(x_{1}, \ldots, x_{k}\right)$ we have $N_{C}(\tau)={ }_{C} \tau$.

Proof. Induction on the structure of the term $\tau$.

Base step. For $\tau \equiv 0,1, \iota, x_{i}$ this is trivial because then $\tau \in T_{0} \subseteq T$ and we take $N_{C}(\tau): \equiv \tau$.

Induction step, by cases according to the type of operations used:

Case 1. Field operations; let $\tau \equiv \tau_{1}+\tau_{2}$. Then $N_{C}\left(\tau_{1}\right) \in T_{n_{1}}$ and $N_{C}\left(\tau_{2}\right) \in T_{n_{2}}$. Take

$$
N_{C}(\tau): \equiv N_{R}\left(N_{C}\left(\tau_{1}\right)+N_{C}\left(\tau_{2}\right)\right) \in T_{\max \left(n_{1}, n_{2}\right)} ;
$$

similarly for the other field operations.

Case 2. Composition operator; let $\tau \equiv \tau_{1} \circ \tau_{2}$.

Case 2.1. For the special case $\tau_{2} \equiv \iota$ we have $\tau_{1} \circ \tau_{2}={ }_{C}$ $N_{C}\left(\tau_{1}\right) \equiv: N_{C}(\tau) \in T$.

Case 2.2. For $\tau_{2} \not \equiv \iota$ we have

$$
N_{C}\left(\tau_{1}\right) \equiv r\left(\iota, v_{1}, \ldots, v_{h}\right) \in N R\left(\iota, v_{1}, \ldots, v_{h}\right) \subseteq T_{n},
$$

and

$$
N_{C}\left(\tau_{2}\right) \equiv s\left(\iota, w_{1}, \ldots, w_{l}\right) \in N R\left(\iota, w_{1}, \ldots, w_{l}\right) \subseteq T_{m} .
$$

Then we get

$$
\begin{aligned}
& N_{C}\left(\tau_{1}\right) \circ N_{C}\left(\tau_{2}\right)={ }_{C} \\
& r\left(\iota, v_{1}, \ldots, v_{h}\right) \circ N_{C}\left(\tau_{2}\right)=C \\
& r\left(N_{C}\left(\tau_{2}\right), v_{1} \circ N_{C}\left(\tau_{2}\right), \ldots, v_{h} \circ N_{C}\left(\tau_{2}\right)\right)={ }_{C} \\
& r\left(s\left(\iota, w_{1}, \ldots, w_{l}\right), v_{1} \circ N_{C}\left(\tau_{2}\right), \ldots, v_{h} \circ N_{C}\left(\tau_{2}\right)\right)={ }_{C} \\
& t\left(\iota, w_{1}, \ldots, w_{l}, v_{1} \circ N_{C}\left(\tau_{2}\right), \ldots, v_{h} \circ N_{C}\left(\tau_{2}\right)\right) .
\end{aligned}
$$

By lemma 3 we see that $v_{1} \circ N_{C}\left(\tau_{2}\right), \ldots, v_{h} \circ N_{C}\left(\tau_{2}\right) \in$ $V_{n+m+1}$. 
Hence if we take, $N_{C}(\tau): \equiv$

$N R\left(t\left(\iota, w_{1}, \ldots, w_{l}, v_{1} \circ N_{C}\left(\tau_{2}\right), \ldots, v_{n} \circ N_{C}\left(\tau_{2}\right)\right)\right)$

we get $N_{C}(\tau) \in T_{n+m+1}$.

Next we want to show, for proving the main theorem later, that $\tau \neq_{C} 0$ follows from $\tau \neq_{R} 0$ for all elements $\tau \in T$. The following examples indicate that we need to construct for every term $\tau \in T$ a term $q \in R(\iota)$ and a constant $a \in Q$ such that $\left.\tau\right|_{x} ^{q} \circ a \neq_{C} 0$. They also show that this is not obvious.

\section{Example 2}

(1) For $v \in V$ we have $v \equiv x_{i} \circ \tau$ or $v \equiv x_{i}$. In both cases we get by substituting $c \in Q, c \neq \neq_{R} 0$, for $x_{i}$ the desired property $\left.v\right|_{x_{i}} ^{c} \neq_{C} c \neq_{C} 0$.

(2) For every term $\tau \in T$ of the form $\tau \equiv t\left(x_{1} \circ\right.$ $\left.\tau_{1}, \ldots, x_{k} \circ \tau_{k}\right)$, where $t \neq_{R} 0$ we get $\tau \neq_{C}$ 0 by substituting suitable constants $c_{1}, \ldots, c_{k}$ for $x_{1}, \ldots, x_{k}$.

(3) For $\tau \equiv x \circ \tau_{1}-x \circ \tau_{2}$ we cannot show that $\tau \neq_{C} 0$ by simply substituting a constant for $x$.

(4) Furthermore, in the case of the term $\tau \equiv(x \circ 1-$ $1) \cdot(x \circ x-x \circ 1)$, we have $\left.\tau\right|_{x} ^{q}={ }_{C} 0$ for $q \equiv c \in Q$ or $q(\iota) \equiv \iota^{n}, n \in \mathbb{N}$.

Lemma 5 For a term $\tau \in T(x)$ of the form $\tau \equiv$ $t\left(\iota, v_{1}, \ldots, v_{l}\right), t \in N R\left(\iota, v_{1}, \ldots, v_{l}\right)$ with $v_{1}, \ldots, v_{l} \in$ $V(x)$ we have the property

$$
t\left(\iota, v_{1}, \ldots, v_{l}\right) \neq_{C} 0 \text { iff } t\left(\iota, v_{1}, \ldots, v_{l}\right) \neq_{R} 0 .
$$

Proof. We construct for every $\tau \in T(x)$ a set of equations $E_{\tau}$ for $q \in R(\iota)$ and a constant $a \in Q$ with the following properties:

(1) if $q$ satisfies the equations $E_{\tau}$ we have $\left.\tau\right|_{x} ^{q} \circ a \neq_{C} 0$;

(2) there always exists a polynomial $q \in R(\iota)$ satisfying the equations $E_{\tau}$.

We introduce now an auxiliary notation for variables. Let $\alpha, \beta$ be fixed symbols. With these we construct sets of variable-symbols as follows:

$$
\begin{aligned}
B_{0} & :=\{\alpha\} \\
B_{n} & :=\left\{\beta_{i} \mid i \equiv t\left(\beta_{i_{1}}, \ldots, \beta_{i_{l}}\right),\right. \\
& \left.t \in N R\left(\beta_{i_{1}}, \ldots, \beta_{i_{l}}\right), \beta_{i_{j}} \in B_{n-1}, j=1, \ldots, l\right\} \\
B & :=\bigcup_{n \in \mathbb{N}} B_{n} .
\end{aligned}
$$

Let $\tau \in T(x), \tau=t\left(\iota, v_{1}, \ldots, v_{l}\right) \in N R\left(\iota, v_{1}, \ldots, v_{l}\right)$ with $t \neq \neq_{R} 0$. There exists a finite set of equations $E_{\tau}$ for $q$, a set of variables $V_{\tau} \subseteq B$, a set of terms $I_{\tau} \subseteq N R\left(V_{\tau}\right)$, and a term $j_{\tau} \in N R\left(V_{\tau}\right)$ with the following properties.
(1) The equations of $E_{\tau}$ are of the form $\beta_{i}={ }_{C} q \circ i$, with $i \in N R\left(V_{\tau}\right)$ and $\beta_{i} \in V_{\tau}$.

(2) For all $i \in N R\left(V_{\tau}\right)$, appearing as argument of $q$ on the r.h.s. of an equation in $E_{\tau}$, we have $i \in I_{\tau}$.

(3) For $j_{\tau}$ we have $j_{\tau} \notin I_{\tau}, j_{\tau} \in N R\left(V_{\tau}\right)$ and $j_{\tau} \neq_{R}$ 0 . Furthermore the following equation holds if all equations of $E_{\tau}$ are satisfied.

$$
j_{\tau}=\left.{ }_{C} \tau\right|_{x} ^{q} \circ \alpha
$$

We construct $E_{\tau}, V_{\tau}, I_{\tau}$ and $j_{\tau}$ inductively. Base step. In the case $\tau \equiv t(\iota, x) \in T_{0}$ take

$$
\begin{gathered}
E_{\tau}:=\left\{\beta_{\alpha}={ }_{C} q \circ \alpha\right\}, \quad V_{\tau}:=\left\{\alpha, \beta_{\alpha}\right\} \\
I_{\tau}:=\{\alpha\}, \quad j_{\tau}: \equiv t\left(\alpha, \beta_{\alpha}\right) .
\end{gathered}
$$

We have to show (3).

$$
\left.t(\iota, x)\right|_{x} ^{q} \circ \alpha={ }_{C} t(\alpha, q \circ \alpha)=_{C} t\left(\alpha, \beta_{\alpha}\right)={ }_{C} j_{\tau} .
$$

For the case $\tau \equiv t(\iota)$ we have $E_{\tau}:=\emptyset, V_{\tau}:=\emptyset, I_{\tau}:=\emptyset$ and $j_{\tau}: \equiv t(\alpha)$.

Induction step. For $\tau \equiv t\left(\iota, x \circ \tau_{1}, \ldots, x \circ \tau_{l}\right)$ with $\tau_{1}, \ldots, \tau_{l} \in T_{n}(x)$ we define

$$
\begin{aligned}
E_{\tau} & :=\bigcup_{m=1}^{l} E_{\tau_{m}} \cup \bigcup_{m=1}^{l}\left\{\beta_{j_{\tau_{m}}}={ }_{C} q \circ j_{\tau_{m}}\right\}, \\
V_{\tau} & :=\bigcup_{m=1}^{l} V_{\tau_{m}} \cup \bigcup_{m=1}^{l}\left\{\beta_{j_{\tau_{m}}}\right\}, \\
I_{\tau} & :=\bigcup_{m=1}^{l} I_{\tau_{m}} \cup \bigcup_{m=1}^{l}\left\{j_{\tau_{m}}\right\}, \\
j_{\tau} & : \equiv t\left(\alpha, \beta_{j_{\tau_{1}}}, \ldots, \beta_{j_{\tau_{l}}}\right) .
\end{aligned}
$$

(1) and (2) are now satisfied by this definition. We want to show (3).

$$
\begin{aligned}
\left.\tau\right|_{x} ^{q} \circ \alpha & ={ }_{C} t\left(\iota,\left.q \circ \tau_{1}\right|_{x} ^{q}, \ldots,\left.q \circ \tau_{l}\right|_{x} ^{q}\right) \circ \alpha={ }_{C} \\
& t\left(\iota \circ \alpha,\left(\left.q \circ \tau_{1}\right|_{x} ^{q}\right) \circ \alpha, \ldots,\left(\left.q \circ \tau_{l}\right|_{x} ^{q}\right) \circ \alpha\right)={ }_{C} \\
& t\left(\alpha, q \circ\left(\left.\tau_{1}\right|_{x} ^{q} \circ \alpha\right), \ldots, q \circ\left(\left.\tau_{l}\right|_{x} ^{q} \circ \alpha\right)\right)={ }_{C} \\
& t\left(\alpha, q \circ j_{\tau_{1}}, \ldots, q \circ j_{\tau_{l}}\right)={ }_{C} \\
& t\left(\alpha, \beta_{j_{\tau_{1}}}, \ldots, \beta_{j_{\tau_{l}}}\right)={ }_{C} j_{\tau} .
\end{aligned}
$$

We used the definitions stated above, the induction hypothesis, the assumption that $q \in R(\iota)$ and repeated application of lemma 2. Furthermore $j_{\tau} \neq_{R} 0$ because $t \neq_{R} 0$. Now we proceed as follows. For $\tau \neq \neq_{R} 0$ construct $E_{\tau}, V_{\tau}, I_{\tau}$ and $j_{\tau}$. Then define

$$
p: \equiv j_{\tau} . \prod_{\{i, j\}, i \neq \neq_{R} j, i, j \in I_{\tau}}(i-j) \in R\left(V_{\tau}\right) .
$$

(Remark: $i \neq_{R} j$ iff $i \not \equiv j$.) With $j_{\tau} \neq_{R} 0$ we see that $p \neq_{R} 0$. Therefore we can find rational constants $b_{i_{1}}, \ldots, b_{i_{k}}, a$ for the variables $\beta_{i_{1}}, \ldots, \beta_{i_{k}}, \alpha \in V_{\tau}$ appearing in $p$ such that $\left.p\right|_{\beta_{i_{1}}, \ldots, \beta_{i_{k}}, \alpha} ^{b_{i_{1}}, \ldots, b_{i_{k}}, a} \neq \neq_{R} 0$. Let $p^{*}$ denote 
$\left.p\right|_{\beta_{i_{1}}, \ldots, \beta_{i_{k}}, \alpha} ^{b_{i_{1}}, \ldots, b_{i_{k}}, a}$. From the construction of the polynomial $p$ it follows that for two terms $i, j \in I_{\tau}$ with $i \neq_{R} j$

$$
i^{*} \neq_{R} j^{*}
$$

Therefore it is easy to construct a polynomial $q \in R(\iota)$ that satisfies the equations

$$
b_{i}={ }_{C} q \circ i^{*}, i \in I_{\tau},
$$

namely

$$
q: \equiv \sum_{i \in I_{\tau}} b_{i} \cdot \prod_{i, j \in I_{\tau}, j \neq f_{R} i} \frac{\left(\iota-i^{*}\right)}{\left(j^{*}-i^{*}\right)} \in R(\iota) .
$$

We get then with (3),

$$
\left.\tau\right|_{x} ^{q} \circ a==_{C} t\left(a, b_{i_{1}}, \ldots, b_{i_{l}}\right)={ }_{C} j_{\tau}^{*} \neq_{R} 0 .
$$

Because of $t\left(a, b_{i_{1}}, \ldots, b_{i_{l}}\right) \in Q$ we conclude $t\left(a, b_{i_{1}}, \ldots, b_{i_{l}}\right) \neq_{C} 0$ and $\left.\tau\right|_{x} ^{q} \circ a \neq_{C} 0$. Since $\left.0\right|_{x} ^{q} \circ a={ }_{C} 0$ we conclude $\tau \neq \neq_{C} 0$, which completes the proof.

Example 3 To illustrate the proof let us compute the polynomial $q$ explicitly for the term given in example 2.4:

$$
\begin{aligned}
\tau & \equiv(x \circ 1-1) \cdot(x \circ x-x \circ 1)={ }_{C} \\
& x \circ 1 \cdot x \circ x-x \circ 1^{2}+x \circ 1-x \circ x \equiv \\
& t(x \circ x, x \circ 1) .
\end{aligned}
$$

For the terms $x$ and 1 we get

$$
\begin{gathered}
E_{x}=\left\{\beta_{\alpha}=C_{C} \circ \alpha\right\}, V_{x}=\left\{\alpha, \beta_{\alpha}\right\}, \\
I_{x}=\{\alpha\}, j_{x} \equiv \beta_{\alpha}, \\
E_{1}=\emptyset, V_{1}=\emptyset, I_{1}=\emptyset, j_{1} \equiv 1 .
\end{gathered}
$$

For $t(x \circ x, x \circ 1)$ we get

$$
\begin{aligned}
& E_{t(x \circ x, x \circ 1)}=\left\{\beta_{\alpha}=_{C} q \circ \alpha, \beta_{\beta_{\alpha}}={ }_{C} q \circ \beta_{\alpha},\right. \\
& \left.\beta_{1}=C q \circ 1\right\}, \\
& V_{t(x \circ x, x \circ 1)}=\left\{\alpha, \beta_{\alpha}, 1, \beta_{\beta_{\alpha}}\right\}, \\
& I_{t(x \circ x, x \circ 1)}=\left\{\alpha, \beta_{\alpha}, 1\right\}, \\
& j_{t(x \circ x, x \circ 1)} \equiv t\left(\beta_{\beta_{\alpha}}, \beta_{1}\right) . \\
& p \equiv t\left(\beta_{\beta_{\alpha}}, \beta_{1}\right) \cdot\left(1-\beta_{\alpha}\right) \cdot(1-\alpha) \cdot\left(\beta_{\alpha}-\alpha\right) \\
& ={ }_{C}\left(\beta_{\beta_{\alpha}} \cdot \beta_{1}-\beta_{1}^{2}+\beta_{1}-\beta_{\beta_{\alpha}}\right) . \\
& \quad\left(1-\beta_{\alpha}\right) \cdot(1-\alpha) \cdot\left(\beta_{\alpha}-\alpha\right) .
\end{aligned}
$$

If we set $a \equiv-1, b_{\alpha} \equiv 0, b_{\beta_{\alpha}} \equiv 1, b_{1} \equiv 2$ we get for $p$ the value $-2 \neq_{R} 0$. For the terms in $I_{\tau}$ we get $\alpha^{*}={ }_{R}-1,1^{*}={ }_{R} 1, \beta_{\alpha}^{*}={ }_{R} 0$ which of course are all different. So the computation yields for $q$

$$
\begin{array}{rl}
q={ }_{C} & 0 \cdot \frac{(\iota-0)(\iota-1)}{(-1-0)(-1-1)}+ \\
& 1 \cdot \frac{(\iota-1)(\iota+1)}{(0-1)(0+1)}+ \\
& 2 \cdot \frac{(\iota-0)(\iota+1)}{(1-0)(1+1)}={ }_{C} \iota+1 .
\end{array}
$$

Finally we want to generalize lemma 5 to the multivariate case.

Lemma 6 For a term $\tau \in T\left(x_{1}, \ldots, x_{k}\right)$ of the form $\tau \equiv t\left(\iota, v_{1}, \ldots, v_{l}\right), t \in N R\left(\iota, v_{1}, \ldots, v_{l}\right)$ with

$v_{1}, \ldots, v_{l} \in V\left(x_{1}, \ldots, x_{k}\right)$ we have the property

$$
t\left(\iota, v_{1}, \ldots, v_{l}\right) \neq_{C} 0 \text { iff } t\left(\iota, v_{1}, \ldots, v_{l}\right) \neq_{R} 0 .
$$

Proof. First we show the fact for the special case of $\tau \in T\left(x_{1}, x_{2}\right)$ with

$$
\tau \equiv t\left(\iota, x_{1} \circ \tau_{1}, \ldots, x_{2} \circ \sigma_{1}, \ldots\right) .
$$

We substitute for $x_{2}$ a term $\rho\left(x_{1}\right)$ such that $\left.x_{2} \circ \sigma_{i}\right|_{x_{2}} ^{\rho\left(x_{1}\right)}$ is not equal to one of the arguments $x_{1} \circ \tau_{j}$. This we can force by taking $\rho\left(x_{1}\right): \equiv x_{1} \circ x_{1} \circ \ldots \circ x_{1}$ (sufficiently often) such that the r.h.s. term never appears as subterm in $x_{1} \circ \tau_{j}$. For the general case we repeat this substitution until we get a term in $T\left(x_{1}\right)$ and then we can apply lemma 5 .

Theorem 1 The function $N_{C}$ is a normal form function for terms in $C$ with respect to the equivalence relation $=_{C}$. For the set of normal forms we have $N C=T$.

Proof. The first property to show for a normal form function is $N_{C}\left(N_{C}(\tau)\right) \equiv N_{C}(\tau)$. This is clear from the construction of $N_{C}$. For the second property assume that $\tau={ }_{C} \sigma$ and $N_{C}(\sigma) \not \equiv N_{C}(\tau)$. We conclude $N_{C}(\sigma) \neq_{R} N_{C}(\tau)$ since $N_{C}$ gives rational normal form. Therefore $N_{C}(\sigma)-N_{C}(\tau) \neq_{R} 0$. By definition of $N_{C}$ we have

$$
N_{C}\left(N_{C}(\sigma)-N_{C}(\tau)\right)={ }_{R} N_{R}\left(N_{C}(\sigma)-N_{C}(\tau)\right) \neq_{R} 0 .
$$

Since $N_{R}\left(N_{C}(\sigma)-N_{C}(\tau)\right) \in T$ we conclude with lemma 6 that $N_{R}\left(N_{C}(\sigma)-N_{C}(\tau)\right) \neq \neq_{C} 0$. On the other hand we conclude from $\tau={ }_{C} \sigma$ with the property $N_{C}(\tau)={ }_{C} \tau$ of lemma 4 that $N_{R}\left(N_{C}(\sigma)-N_{C}(\tau)\right)={ }_{C} 0$ which results in a contradiction. For the set of normal forms remark that if $\sigma \in T$ there is $N_{C}(\sigma) \equiv \sigma$ and so $N C=T$.

Remark. The size of the normal form can grow exponentially with the size of the input. Consider for example the term

$$
(\sigma+\tau) \circ(\sigma+\tau) \circ \ldots \circ(\sigma+\tau) \text {. (see below) }
$$




\section{Implementation}

The implementation of the algorithm in mathematica is given by the following compact rule-based program, using the full power of the pattern-matching abilities of mathematica [7].

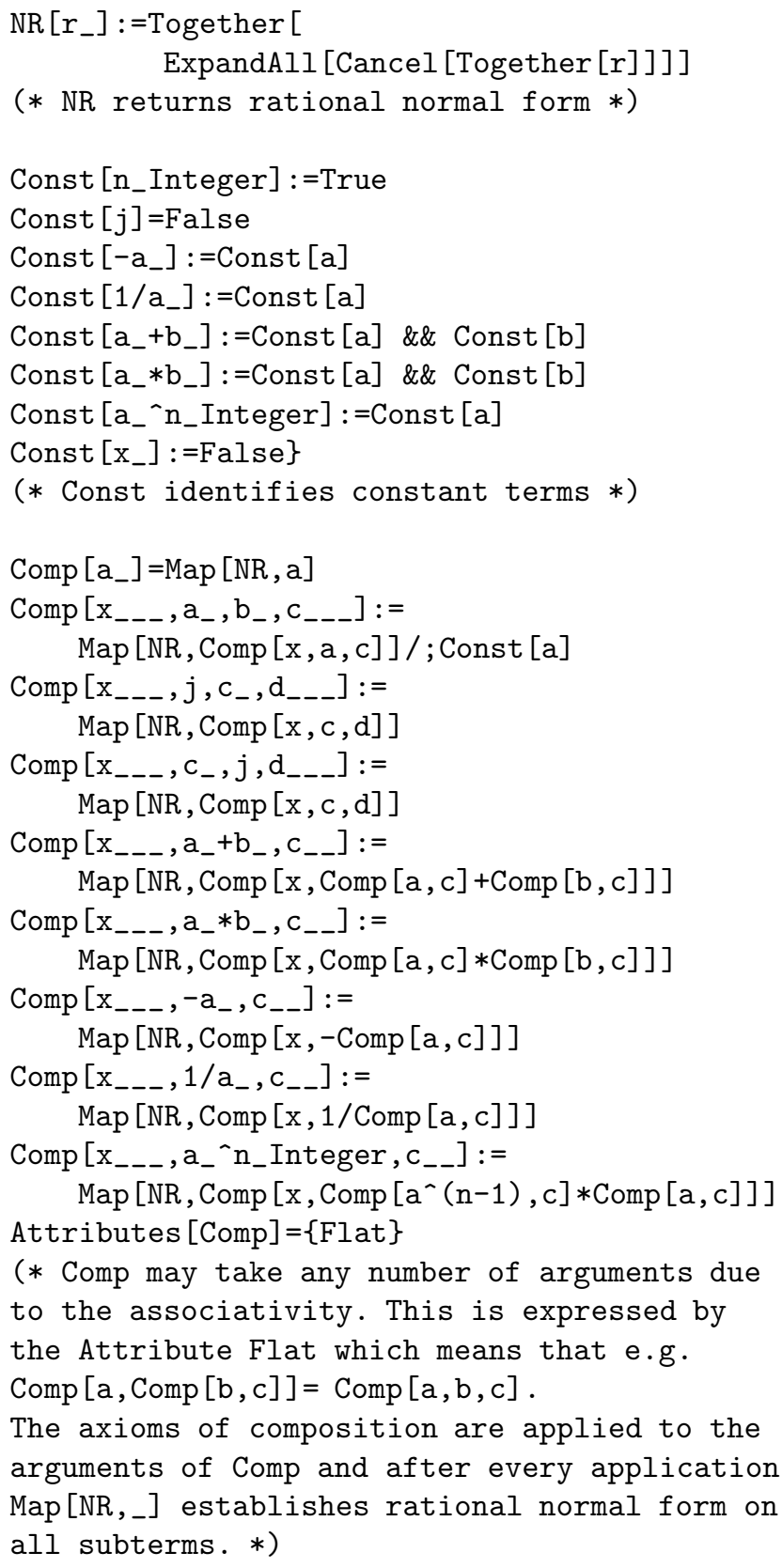

The first example given in the introduction is handled by this program as follows.

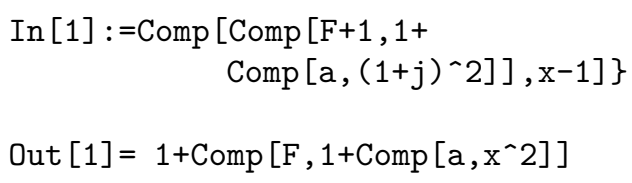

Now let us compute the complicated expression from the introduction.

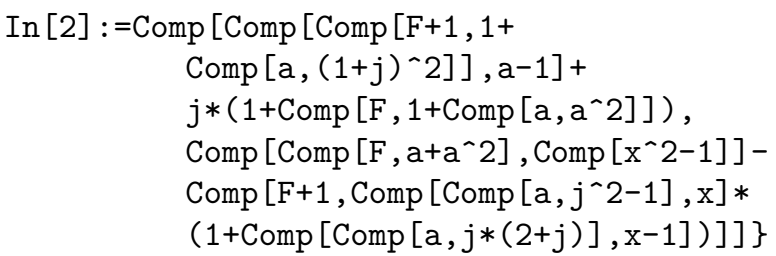

Out $[2]=0$

As illustration of the last remark in the previous chapter we compute.

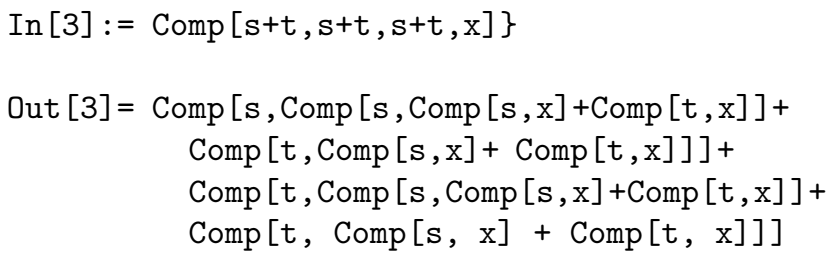

\section{Concluding Remarks}

A natural interpretation for composition terms are programs built up by rational operations and function calls. For example the two terms of our first introducing example could be interpreted as the mathematical representations of the following two programs. The first term representing the computation of $f(x)$ :

$$
\begin{aligned}
& \text { function } f(x) ; \\
& f:=x-1 ; \\
& f:=h(f) ; \\
& \text { return }(f) ; \\
& \text { function } h(x) ; \\
& h:=(x+1)^{2} ; \\
& h:=1+a(h) ; \\
& h:=l(h) ; \\
& \text { return }(h) ; \\
& \text { function } l(x) ; \\
& l:=F(x) ; \\
& l:=l+1 ; \\
& \text { return }(l) ;
\end{aligned}
$$

The second term representing the computation of $g(x)$ :

$$
\begin{aligned}
& \text { function } g(x) ; \\
& g:=x^{2} ; \\
& g:=1+a(g) ; \\
& g:=F(g) ; \\
& g:=g+1 ; \\
& \text { return }(g) ;
\end{aligned}
$$

These two programs, using unknown subprograms for computing $a(x)$ and $F(x)$, will compute the same results for all $a(x), F(x)$ and inputs $x$. Now we are not only in the position to tell whether programs of the kind above 
always compute the same results, but furthermore we can, when they are different, give by lemma 5 and 6 explicitely instances of the unknown subprograms such that this is the case.

A natural extension of the algorithm would be to the case of multivariate functions. Another extensions could be made by introducing additional functions into the theory by new axioms like exp $\circ(x+y)=$ $(\exp \circ x) \cdot(\exp \circ y)$.

When interested in the rewriting approach [5], it could be worth to restrict to rings, since it is difficult to see how to do the computation of normal forms in fields, classically based on GCD-computation, by rewriting techniques. In rings Knuth-Bendix-algorithms for computing the normal form are known. On the other hand a pure compositional structure is a monoid also allowing normal form computation by a Knuth-Bendixalgorithm. Therefore it seems quite plausible to extend the Knuth-Bendix-algorithm for rings to a normal form algorithm for function rings, involving rules similar to those in the mathematica program given above. In this framework the composition axiom for constants could serve as a test case for conditional term rewriting.

\section{Acknowledgements}

I wish to thank Prof. E.Engeler for his encouragement and help in improving the paper, Oliver Gloor for carefully reading the paper, and an anonymous referee for his knowledgeable comments.

\section{References}

[1] Aberer, K.: "Normal Forms in Combinatory Differential Fields". ETH-Report No. 89-01, (1989).

[2] Davenport, J.H., Siret, Y. and Tournier, E.: "Computer Algebra". Academic Press, N.Y., (1988).

[3] Engeler, E.: "Combinatory Differential Fields". to appear in Theoretical Computer Science, (1990).

[4] Geddes, K.O., Labahn, G., Czapor, S.R.: "Algorithms for Computer Algebra". preprint, (1989).

[5] Le Chenadec, P.: "Canonical Forms in Finitely Presented Algebras", Research Notes in Theoretical Computer Science, Pitman, (1986)

[6] Menger, K.: "Function Algebra and Propositional Calculus". Self-Organizing Systems, Spartan Books, (1962), p. 525ff.

[7] Wolfram, S.: "Mathematica". Addison-Wesley Publishing Company, (1988). 